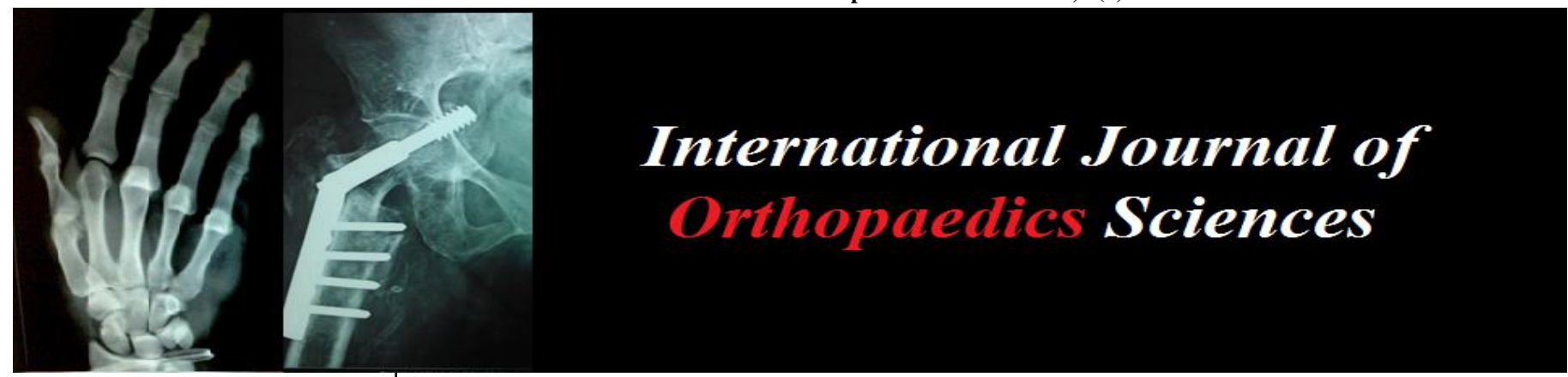

ISSN: $2395-1958$

IJOS 2018; 4(2): 80-83

(C) 2018 IJOS

www.orthopaper.com

Received: 11-02-2018

Accepted: 12-03-2018

Dr. Rahul Singh

Assistant Professor, Department of Orthopedics, Mayo Institute

of Medical Sciences, Barabanki,

Uttar Pradesh, India

Dr. Rajendra Goyal

Consultant orthopedic Surgeon,

Jindal Hospital, Bharatpur,

Rajasthan, India

\section{Functional and radiological outcome of unstable distal radius fracture treated by conservative or volar buttress plate}

\section{Dr. Rahul Singh and Dr. Rajendra Goyal}

DOI: https://doi.org/10.22271/ortho.2018.v4.i2b.11

\section{Abstract}

Treatment of distal radius fracture is controversial; there is no single definitive treatment method that is considered the standard of care. It is important today to determine the nature of the fracture and to describe the bio-mechanism involved than to link diagnosis and treatment. This study involved total 50 patients, 18 cases were operated 32 were managed conservatively. Treatment to be received was decided by the patients themselves after thorough counseling about pros \& cons of each modality, according to their financial status, activity level and associated comorbidities. Residual deformity PUS, RDT and RD in operative group were $11.11 \%, 5.55 \%$ and $11.11 \%$ respectively while in conservative group PUS, RDT and $\mathrm{RD}$ were $12.5 \%, 22.50 \%$ and $25.00 \%$ respectively.

Keywords: Functional and radiological outcome, unstable distal radius fracture, volar buttress plate

\section{Introduction}

Impacted infra-articular fractures have generated interest recently because the failure to reduce these fractures to within $2 \mathrm{~mm}$ of articular congruity particularly in young adults has been shown to lead to symptomatic post traumatic arthritis. Knirk and Jupiter (1986) found that it this fracture healed with greater than $2 \mathrm{~mm}$ of residual articular incongruity $100 \%$ of these patient had radiographic evidence of arthritis and two thirds were symptomatic.

Treatment of distal radius fracture is controversial; there is no single definitive treatment method that is considered the standard of care. It is important today to determine the nature of the fracture and to describe the bio-mechanism involved than to link diagnosis and treatment. The principles of treatment for distal radius fractures are the same as those for any other articular or periarticular fracture: anatomic reconstruction, stable fixation, and early motion.

Closed reduction and plaster casting is still the treatment of choice most fractures of distal radius especially stable ones or unstable also. Fractures of the distal radius are not to be underestimated because even though an anatomical reduction is achieved as a good result, but there is great deal of secondary displacement, gross radiological abnormalities are significantly associated with fair to poor results. It appears that improved anatomical results combined with early rehabilitation of wrist function provide very favourable results. Maintaining the reduced position of a unstable fracture however remains a problem. Plaster has been shown to be ineffective (Stewart et al. 1984) ${ }^{[1,2]}$

The treatment of Colle's fractures by triple intra focal wire fixation advocated by I.A. Kapandaji in triple pinning (1987), double pinning (1973) had in view to satisfy three main requirements i. e. to obtain a bony fusion in good position so that rehabilitation can be started immediately to avoid trophic changes and the effects of disuse and to allow a rapid return to work and leisure.

Many now feel that the external fixation technique has replaced pins and plaster as the treatment for most unstable distal radius fractures (Seitz et al. 1991). There is also more focus on biomechanical aspects of distraction to determine excessive traction, as it is associated with higher complications (Kaempffe 1993, Winemaker 1998, Davenport 1999) and effect of distraction on carpal kinematics (Ishikawa 1999) ${ }^{[3]}$.
Correspondence

Consultant orthopedic Surgeon, Jindal Hospital, Bharatpur,

Rajasthan, India 
There has been a new concept in the management of complex fractures called augmented external fixation, where in addition to external fixation, limited internal fixation (with kwires), limited Open reduction bone grafting (cementation) or open reduction and internal fixation are used in combination to restore radial anatomy including articular congruity (Axeirod 1988, Sietz 1991, Jupitor 1901, Putnam 1997, Zaimotti 1997, Wolfe 1998 \& 1999). All have shown good results ${ }^{[4]}$

External fixation for the distal radius, however frequently fail to provide sufficient stability for early motion, which may be a critical factor in functional outcome. Still open reduction and internal fixation hasyet to replace external fixation completely, with or without adjunctive K-wire fixation, as the most common method of treatment for unstable distal radius fractures. Early motion of the wrist has been advocated not only to decrease the stiffness associated with prolonged immobilization but also to stimulate cartilage repair, to assist the so-called moulding of articular fragments, and to diminish the development of periarticular osteopenia.

Many new plates including dorsal, low-profile, volar, and/or uniquely designed plates recently have been developed for internal fixation of distal radius fracture. ${ }^{5}$

Although there is a dearth of strict prospective, randomized studies or biomechanical data indicating clear superiority of one treatment modality over another, there continues to be a strong push toward anatomical reduction and internal especially on the volar side with use of angular stable fixation. It is remarkable that this trend toward internal fixation is so strongly driven without clear scientific evidence supporting it.

Many things are subject to trend and fashion, and the fixation treatment of distal radial fractures is no exception. Pins and plaster gave way to external fixation, and now internal fixation has begun to supplant all other treatment modalities. Novel fields of research, epidemiologic and sociologic changes, and new implant, designs are driving this new trend toward plate fixation.

\section{Methodology}

This study involved total 50 patients, 18 cases were operated 32 were managed conservatively. Treatment to be received was decided by the patients themselves after thorough counseling about pros \& cons of each modality, according to their financial status, activity level and associated comorbidities.

\section{Techniques}

(a) Closed reduction \& cast: We after achieving adequate analgesia, applied in-line traction with assistant facilitate relaxation of the forearm musculature. Required manipulation will depend on the presenting fracture, once the fracture fragments are disim pacted, each component of the fracture displacement reduced according to fracture $\&$ for plaster immobilization we tried to adapt the position of the hand and wrist to a position that is directly opposite to the displacement that occurred in producing the original deformity and slab applied such as for the typical dorsally angulated fracture with minimal displacement of the volar cortex, the reduction can be obtained by direct pressure on the distal fragment from the dorsal surface to correct the angulation.

Translation or "unlocking" of the volar cortex if overlapped. This is followed by direct pressure on the distal fragment to correct the angulation. Longitudinal traction with palmar flexion of the wrist effects a reduction of the fracture. The volar and dorsal rim of the radius can be palpated to give an estimate of the correction of volar tilt before slab application. Check Xray done \& discharged for 1-2 week. The length of immobilization varies from 4 to 6 weeks.

(b) Open reduction and internal fixation: Brachial plexus block given \& position on OT table. Scrubbed, tourniquet on arm applied, painted \& draped. An $8-10 \mathrm{~cm}$ incision is made on the radial border of the flexor carpi radialis tendon. The incision is carried through the flexor carpi radialis tendon sheath. The tendon sheath is opened and the forearm fascia on the radial border of the flexor carpi radialis is incised. The forearm fascia incision should be madeb long the radial side of the flexor carpi radialis tendon to ensure that the palmar cutaneous branch of the median nerve, which arises near the ulnar side of the flexor carpi radialis tendon is protected. The index finger is then swept under the flexor pollicis longus muscle to gain rapid exposure of the pronator quadratus. The attachment of the flexor pollicis longus to the radius is incised and partially detached for a full view of the Pronator quadratus after the placement of retractors. A Lshaped incision is made over the radial border of the pronator quadratus to prevent full elevation of that structure from the radius. The pronator quadratus can be reattached after plate fixation by sewing it to the brachioradialis, which inserts on the radial styloid. After full exposure of the fracture site the hematoma and fibrinous material are removed from the fracture site. For ease of fracture reduction, a elevator or an osteotome is inserted into the fracture, over the dorsal cortex, to completely dis impact the distal fracture fragment. Fracture reduction is facilitated through ligamentotaxis by applying traction to the fingers. The assistant distracts the fracture while the surgeon reduces it through manual manipulation.

For dorsal comminuted fractures, the surgeon can use manual pressure to mold the dorsal fragments into anatomical reduction. When the distal fracture fragment is being reduced into anatomic position, it is important for an assistant to apply traction continuously to the fingers of the operatively treated hand. In comminuted impacted fractures, the distal fragment (s) must be dis impacted sufficiently such that avoid at the fracture line is often seen. For displaced radial styloid fractures, a number-15 knife blade is swept along the radial border of the radius under the brachioradialis to partially release its insertion and relieve the deforming force on the radial styloid. Finally, screw is placed in each of the proximal holes. Intraoperative radiographs should be examined to ascertain that the plate is in the optimal position \& sutured to the edge of the brachioradialis with use of absorbable horizontal mattress sutures to provide coverage for the distal plate this will prevent irritation of the flexor tendons by the plate. The wound is then closed with use of absorbable materress sutures.

\section{Results}

The most common complication encountered was finger stiffness affecting $3(16.66 \%)$ patients and $8(25 \%)$ patients of operative \& conservative group respectively. At 6 month follow Up the finger stiffness complication was remain in $3(11.11 \%)$ in Conservative group. It shows the finger stiffness complication at 6 month follow up was disappear in patients of Operative group as compare to Conservative group. 
Second most common complication observed was pain DRUJ affecting $11 \%$ \& $22 \%$ patients in operative \& conservative group which again decrease to $0 \%$ and $7 \%$ at 6 months. At 6 months the complication taking predominance was arthritic changes at radio carpal joint affecting $9 \%$ \& $11 \%$ patient respectively.

After procedure the mean $\pm \mathrm{SD}$ of $\mathrm{RL}$ were $11.33 \pm 2.00$ in operative group while in conservative group is 9.7513 .41 of the affected hand. The velar tilt or dorsal angle mean \pm SD were 6.6113 .76 and. $37 \pm 9.92$ in operative group and conservative group respectively after the procedure. The $3^{\text {rd }}$ parameter radial angle means $\mathrm{SD}$ is 19.4414 .70 and $18.43 \pm 4.65$ in operative group and conservative group respectively after the procedure

Residual deformity PUS, RDT and RD in operative group were $11.11 \%, 5.55 \%$ and $11.11 \%$ respectively while in conservative group PUS, RDT and RD were $12.5 \%, 22.50 \%$ and $25.00 \%$ respectively

Table 1: Distribution of Comminution and Associated Injury

\begin{tabular}{|c|c|c|c|c|c|}
\hline \multirow{2}{*}{ Particulars } & \multicolumn{3}{|c|}{ Comminution } & \multicolumn{2}{c|}{ Associated Injury } \\
\cline { 2 - 6 } & Intra-Articular & Extra-Aricular & Total & \# Ulna or Styloid & \# other Bone or system \\
\hline \multirow{2}{*}{ OP } & 13 & 5 & 18 & 11 & 8 \\
& $(72.22)$ & $(27.78)$ & $(100.0)$ & $(61.11)$ & $(44.44)$ \\
\hline \multirow{2}{*}{ CRS } & 26 & 6 & 32 & 5 & 0 \\
& $(81.25)$ & $(18.75)$ & $(100.00)$ & $(13.18)$ & $(0.00)$ \\
\hline \multirow{2}{*}{ Total } & 39 & 11 & 50 & 38 & 8 \\
& $(78.00)$ & $(22.00)$ & $(100.0)$ & $(76.00)$ & $(16.00)$ \\
\hline
\end{tabular}

Table 2: Mean \pm SD of initial radiological parameters

\begin{tabular}{|c|c|c|c|c|}
\hline \multirow{2}{*}{ Parameters } & \multicolumn{2}{|c|}{ OP } & \multicolumn{2}{c|}{ CRS } \\
\cline { 2 - 5 } & Affected & Normal & Affected & Normal \\
\hline RL & $3.54 \pm 3.00$ & $13.22 \pm 1.27$ & $4.06 \pm 4.59$ & $12.69 \pm 1.18$ \\
\hline VT/DA & $-7.00 \pm 13.04$ & $9.78 \pm 1.31$ & $-6.25 \pm 16.85$ & $9.68 \pm 1.13$ \\
\hline RA & $7.56 \pm 6.28$ & $23.72 \pm 2.40$ & $11.72 \pm 8.61$ & $24.44 \pm 2.42$ \\
\hline
\end{tabular}

Table 3: Mean \pm SD of postoperative radiological parameters

\begin{tabular}{|c|c|c|c|c|}
\hline \multirow{2}{*}{ Parameters } & \multicolumn{2}{|c|}{ OP } & \multicolumn{2}{c|}{ CRS } \\
\cline { 2 - 5 } & Affected & Normal & Affected & Normal \\
\hline RL & $11.33 \pm 2.00$ & $13.22 \pm 1.27$ & $9.75 \pm 3.14$ & $12.69 \pm 1.18$ \\
\hline VT/DA & $6.61 \pm 3.76$ & $9.78 \pm 1.31$ & $1.37 \pm 9.92$ & $9.68 \pm 1.13$ \\
\hline RA & $19.44 \pm 4.70$ & $23.72 \pm 2.40$ & $18.43 \pm 4.65$ & $24.44 \pm 2.42$ \\
\hline
\end{tabular}

Table 4: Distribution of Residual Deformity

\begin{tabular}{|c|c|c|c|c|}
\hline \multirow{2}{*}{ Particulars } & \multicolumn{2}{|c|}{ OP } & \multicolumn{2}{c|}{ CRS } \\
\cline { 2 - 5 } & No. & \% & No. & \% \\
\hline PUS & 2 & 11.11 & 4 & 12.50 \\
\hline RDT & 1 & 5.55 & 7 & 22.50 \\
\hline RD & 1 & 5.55 & 8 & 25.00 \\
\hline
\end{tabular}

Table 5: Functional Result According To Garland and Werley Criteria

\begin{tabular}{|c|c|c|c|c|}
\hline \multirow{2}{*}{ Outcome } & \multicolumn{2}{|c|}{ At 3 months } & \multicolumn{2}{c|}{ At 6 months } \\
\cline { 2 - 5 } & OP & CRS & OP & CRS \\
\hline \multirow{2}{*}{ Excellent } & 7 & 0 & 11 & 13 \\
& $(38.89)$ & $(0.00)$ & $(100.0)$ & $(48.15)$ \\
\hline \multirow{2}{*}{ Good } & 10 & 9 & 0 & 12 \\
& $(55.55)$ & $(28.12)$ & $(0.00)$ & $(44.44)$ \\
\hline \multirow{2}{*}{ Fair } & 1 & 20 & 0 & 2 \\
& $(5.56)$ & $(62.50)$ & $(0.00)$ & $(7.41)$ \\
\hline \multirow{2}{*}{ Poor } & 0 & 3 & 0 & 0 \\
& $(0.00)$ & $(9.38)$ & $(0.00)$ & $(0.00)$ \\
\hline \multirow{2}{*}{ Total } & 18 & 32 & 11 & 27 \\
& $(100.00)$ & $(100.0)$ & $(100.0)$ & $(100.0)$ \\
\hline
\end{tabular}

\section{Discussion}

A short-arm cast is sufficient for immobilization, demonstrating equivalent radiographic and functional outcomes in adult populations when compared with aboveelbow immobilization\&. Non circumferential immobilization is appropriate when swelling, potential compartment syndrome.

Wahl storm (1983) demonstrated by bone scanning that unstable fractures are healed and stable after 4 weeks and do not immobilization longer than 4 weeks. The aims of conservative treatment are to obtain and maintain anatomic realignment of the fracture for a period of 6 weeks, the time at which bony union is well advanced, so that the risk for secondary displacement at that stage is minimal. In our study we applied below elbow slab for 6 to 8 weeks according to fracture pattern and tightened the slab at every follow up period up to healing ${ }^{[6]}$.

Despite an adequate initial reduction and immobilization, redisplacement is common, caused by deforming forces across the wrist as well as individual patient factors. Significant indirect deforming forces across the fracture include the long flexor and extensor tendons. Deforming forces directly influencing fracture fragments include the brachioradialis, which has a consistent pattern of insertion on the radial side of the styloid and may contribute to the secondary displacement of this fragment. Adequately reduced distal radius fractures require follow-up radiographs to assess for re-displacement and healing ${ }^{[7]}$.

Loss of reduction most commonly occurs during the second week of cast treatment (Hove LM 1994, Altissimim 1994) or later (Diss JJ 1987), especially in the elderly. Our study supported these studies because in our study 6 cases of unstable fracture initially were selected conservative modality of treatment $\&$ acceptable reduction achieved but follow up between 2 to 6 week checkup X-ray were found displaced \& malunited and 4 out of 6 were young and two were old in fact it indicate conservative treatment failure. Five cases out of six were turned over to operative modality and were excluded from conservative group because the duration of follow up these patients were shorter so functional result evaluation not possible ${ }^{[8]}$. 
This suggests that the timing and number of follow-up visits needs to be individualized to both the patient and fracture. For those fractures that are unstable, immobilization to at least 6 weeks is indicated (Diss JJ 1987, Hove 1994). When a loss of reduction is observed, a repeat reduction may be attempted; however, Schmalholz (1989) demonstrated that a recurrence of deformity is common.

Difficulty obtaining adequate correction of intra-articular displacement and maintaining axial stability throughout the course of treatment are the two major limitations of nonoperative management. Successful nonoperative treatment requires careful patient selection, an initial demonstrated to improve reduction rates or long term outcomes (Earnshow 2002) ${ }^{[9,10]}$.

Despite the large body of literature on the subject, results of the non-operative management of distal radius fractures remain conflicted. A recent Cochrane review has found insufficient evidence to recommend any particular method of reduction, or duration and type of immobilization for distal radial fractures (Hondall HH 2003). It is clear that in most instances, restoration of the normal anatomy is important for the restoration of function, and thus patient satisfaction (Chang 2001). Radiographic factors predictive of instability may also be linked to function, with several studies reporting that the preservation of radial length was most important for the preservation of function (Warwick D 19931 followed by palmar angulation (Tsukazaki T 19931).

\section{Conclusion:}

In our study according to Stewart scoring system result obtained in conservative group 9 patients $(30 \%)$ had excellent, 5 patients (17\%)-had good result and 16 patients $(53 \%)$ had fair anatomical reduction result.

\section{References}

1. Conney WP, Linschied RL, Dobyns JH. External pin fixation for unstable colles fractures. J Bone Joint Surg. 1976; 61A:840-845.

2. Vesely DG, Conwel I 1 a, Fractures of the distal radius in adults. Clin Orthop. 1972; 83:13-16.

3. Corey Trease. Locking versus non locking $\mathrm{T}$ plate for dorsal and volar fixation of dorsally communited distal radial fractures; a biomechanical study; The Journal of Hand Surgery. 2006; 30(4)756-762

4. Cylbum TA. Dynamic external fixation for comminuted intra articular fractures of the distal end of the radius. J Bone Joint Surg, 1987; 69A:248-254.

5. Wright TW, Davenport WC, Garymiller, Gainesville FL. Wrist ligament strain during external fixation. J Hand Surg. 1999; 24A:102-107.

6. David Ringl. Combined dorsal and volar plate fixation of complex fracture of the distal part of the radius. 2005; 87A(1-2):195-212

7. De Palma AF. Comminuted fractures of the distal end of the radius treatment by ulnar pinning. J Bone Joint Surg. 1952; 34:651-662.

8. Dias JJ, Wray CC, Jones JM, Gregg P1. The value of early mobilisation in the treatment of Colles fractures, $\mathrm{J}$ Bone Joint Surg. 1987; 69B:463-467.

9. Sawyer B, Dowling JJ. Comminuted Colles fractures: Evaluation of a method of treatment. J Bone Joint Surg. 1961; 43A: 657-668.

10. Ehsan A, Stevanovic M. Skeletally mature patients with bilateral distal radius fractures have more associated injuries. Clin Orthop Relat Res, 2009. 